\title{
Validation of the CUTLASS HF radar gravity wave observing capability using EISCAT CP-1 data
}

\author{
N. F. Arnold, T. B. Jones, T. R. Robinson, A. J. Stocker, and J. A. Davies \\ Department of Physics and Astronomy, University of Leicester, University Road, Leicester, LE1 7RH, United Kingdom
}

Received: 22 September 1997 / Revised: 27 April 1998 / Accepted: 6 May 1998

\begin{abstract}
Quasi-periodic fluctuations in the returned ground-scatter power from the SuperDARN HF radars have been linked to the passage of medium-scale gravity waves. We have applied a technique that extracts the first radar range returns from the F-region to study the spatial extent and characteristics of these waves in the CUTLASS field-of-view. Some ray tracing was carried out to test the applicability of this method. The EISCAT radar facility at Tromsø is well within the CUTLASS field-of-view for these waves and provides a unique opportunity to assess independently the ability of the HF radars to derive gravity wave information. Results from 1st March, 1995, where the EISCAT UHF radar was operating in its $\mathrm{CP}-1$ mode, demonstrate that the radars were in good agreement, especially if one selects the electron density variations measured by EISCAT at around $235 \mathrm{~km}$. CUTLASS and EISCAT gravity wave observations complement each other; the former extends the spatial field of view considerably, whilst the latter provides detailed vertical information about a range of ionospheric parameters.
\end{abstract}

Key words: Ionosphere (ionosphere - atmosphere interactions) - Meteorology and atmospheric dynamics (thermospheric dynamics) - Radio science (ionospheric propagations)

\section{Introduction}

Since the seminal work of Hines (1960), gravity waves have been recognised as an important phenomenon in the upper atmosphere (see Hunsucker, 1982; Hocke and Schlegel, 1996, for reviews). They are able to transport

Correspondence to: N. F. Arnold energy and momentum from the middle atmosphere and from the auroral regions to mid-latitudes (Chimonas and Hines, 1970). Whilst periodic fluctuations in the auroral current can induce gravity waves (e.g. Millward, 1994), single pulse disturbances need to undergo dispersive reflection from the Earth's surface to exhibit wavelike properties (Francis, 1974). Periodic waves may also be generated by instability processes associated with convection electric fields in the auroral F-region (Robinson, 1994).

Much of our knowledge of thermospheric gravity waves comes indirectly from studying the induced response of the ionosphere (e.g. Munro, 1958; Georges, 1968). Often this work was carried out using vertical HF radio transmitter/receiver pairs such as ionosondes and Doppler sounders. However, the relationship between the waves and the ionosphere is not a simple one and quite sophisticated inversion techniques have been adopted to obtain wave parameters from ionospheric measurements (Kirchengast et al., 1995, 1996). A useful test of the ionospheric disturbances' similarity to the underlying gravity wave field comes from applying Hines' (1960) dispersion relationship for the neutral waves. Reasonable agreement between the two indicated a broadly linear response (e.g. Tedd and Morgan, 1985).

Samson et al. (1990) have demonstrated that travelling ionospheric disturbances (TIDs) associated with atmospheric gravity waves can be detected from the modulations in the power and Doppler shifts of the ground-backscatter returns from oblique HF radars. Whilst this has allowed a determination of the period of the waves and the direction of the sources, fluctuations in the signal strength have hampered attempts to obtain the amplitude of the disturbances using this technique.

We shall present results from the Collaborative United Kingdom Twin Located Auroral Sounding System (CUTLASS) radar facilities in Iceland and Finland which can provide information about the period, azimuth and amplitude of the travelling ionospheric disturbances related to atmospheric gravity waves. We employ a new technique which makes use 
of the 'first skip' radar propagation path which is relatively insensitive to fluctuations in backscattered power. As part of the validation process, a comparison with the European Incoherent Scatter (EISCAT) UHF radar was made. The EISCAT facility has been in operation for around fifteen years (see, e.g., Baron, 1984) and has been used successfully to study a number of gravity wave properties (Schlegel, 1986; Natorf et al., 1992; Lanchester et al., 1993; Shibata and Schlegel, 1993; Williams et al., 1993). Further refinements of the first skip technique will be discussed.

\section{2 'First skip' theory}

The problem of range determination using radars is an old one (see, e.g., Gething, 1991 for a review). If the radar waves propagated in straight lines and the ionosphere behaved like a smooth reflecting surface, then the true distance of a target on the ground could be obtained from simple trigonometry. Modern ray-tracing techniques have taken account of the continuous variations in the refractive index of the ionosphere to produce a more accurate estimate of the range, but temporal and spatial variations in the medium remain (Tedd et al., 1984). Gravity waves are able to distort the apparent position of a target by modifying the reflection angle of the ray path. A combination of ray-tracing and a detailed knowledge of the current state of the ionosphere would be needed to get a complete picture of the variation in the first returns from the F-layer.

In simple 'tilting mirror' theory, the apparent range of an object will change depending on the angle of the ionosphere presented to the rays. If the ray path is fairly oblique, then relatively small tilts will produce significant variations in the range. Assume a perfect reflecting mirror and monochromatic waves such that

$A=A_{0} \sin (k x-\omega t+\phi)$

where $A_{0}$ is the amplitude of the wave, $k$ is the wave number, $\omega$ is the angular velocity and $\phi$ is the phase. Then the gradient is given by

$\frac{\mathrm{d} A}{\mathrm{~d} x}=k \cdot A_{0} \cos (k x-\omega t+\phi)$.

The range as a function of tilt angle can be given from the following equations:

$\Delta_{\mathrm{R}}=\tan ^{-1}\left(h / D_{1}\right)$

$\Delta_{\mathrm{T}}=\tan \left(\Delta_{\mathrm{R}}-2 \theta_{\text {tilt }}\right)$

$D_{2}=h / \tan \left(\Delta_{\mathrm{T}}\right)$

where $h$ is the vertical distance to the reflecting layer, $D_{1}$ is the horizontal distance from the radar to the reflection point, $D_{2}$ is the horizontal distance from the reflection point to the ground scatter region and $\theta_{\text {tilt }}$ is the gradient of the reflecting layer with respect to the undisturbed ionosphere. $\Delta_{\mathrm{R}}$ and $\Delta_{\mathrm{T}}$ are the elevation angles of the transmitted/received wave and the ground-scatter angle respectively. See Fig. 1 for a schematic view of the geometrical arrangements.

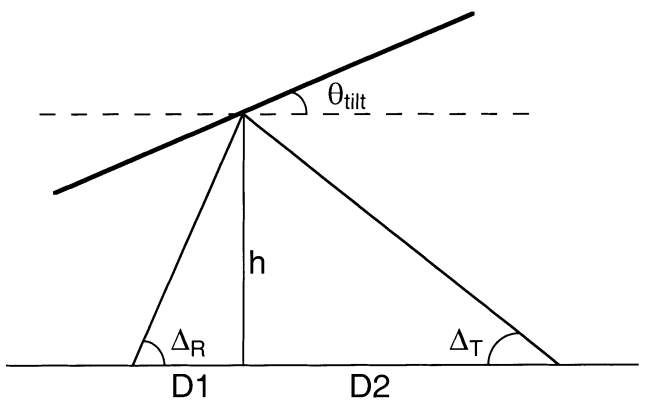

Fig. 1. Schematic of an idealised ionospheric 'tilting' reflecting layer

The oblique angle of the CUTLASS radars ensures that relatively small variations in the effective vertical reflection height can result in significant changes in the horizontal skip distance. It should be noted however that the response tends to be non-linear. The mean skip distance will tend to increase away from the radar and a monochromatic wave will generate higher frequency components in the skip which must not be confused with genuine non-linearity in the ionospheric response to the passage of a gravity wave (see, e.g., Arnold and Robinson, 1994).

\section{The CUTLASS Iceland and Finland radars}

Located at sites in Hankasalmi in central Finland and Pykkvibaer in Iceland, the CUTLASS radars each comprise of a linear array of 16 antennas fed by a distributed system of transmitters/receivers. The design closely follows that of a number of other radars that form part of the SuperDARN observing network, deployed to observe the auroral region (Greenwald et al., 1995). The radars can operate over a range of HF frequencies varying from $8-20 \mathrm{MHz}$. The antenna area produces a beam with a main lobe $4^{\circ}$ wide and side lobe power down by over a factor of 10 . Electronic phase delays can be imposed on the different antennas to rotate the direction of the beam by up to $45^{\circ}$.

A coded pulse sequence is transmitted and autocorrelation functions of the received signal provide information about the power, Doppler velocity and spectral width. Range data can be inferred with a resolution of $45 \mathrm{~km}$ for 70 range gates out to approximately $3000 \mathrm{~km}$. The integration time for the standard operational mode is seven seconds, allowing a complete scan of the 16 viewing positions every $2 \mathrm{~min}$. This is sufficiently below the Nyquist limit to permit studying gravity waves with periods of $15 \mathrm{~min}$ or more. The signal-to-noise ratios are high enough for an integration period of one second, but this sampling rate would only become necessary when more than one complete scan at a range of frequencies was required.

Samson et al. $(1989,1990)$ have interpreted wave-like modulations in the ground-scatter power returns as being due to the focusing and defocusing of the radio waves by the passage of atmospheric gravity waves. Bristow et al. (1994) and Bristow and Greenwald (1995) 
have computed the atmospheric gravity wave spectra using 2-h samples of the power return data at each range-beam element. They relied on the power variation of the gravity wave modulated ground scatter data. However, this data is sensitive to changes in the ionospheric propagation conditions that affect the signal strength. In addition, there are a few days each year when the ionospheric conditions are such that strong ground-scatter returns over an extended number of radar ranges are quite rare. Our technique provides complementary evidence that is less sensitive to absolute power fluctuations and can be applied when good observations can be obtained for a limited number of range gates. Ground-scattered power has been identified from the very low Doppler shifts present during the measurements (absolute values less than $50 \mathrm{~ms}^{-1}$ ). Similar patterns were obtained for the other beams, indicating that the disturbances were of a spatially extended nature, consistent with a propagating wave front. As the time of arrival of the phase front was delayed with respect to the nearer range gates, one can infer that the waves were travelling towards the radar.

Careful inspection of the phase fronts of the earliest radar returns from the F-region, starting around 05:00 UT and continuing up to 08:00 UT in Fig. 2, and the latest starting around 15:00 UT, indicate that these waves were travelling away from the radar. This apparent anomaly was resolved by Milan et al. (1997) who proposed that when the forward-directed beams are looking at the ionosphere when it is still in darkness, the backward-looking side lobes are able to receive signals propagating via the sunlit ionosphere at lower latitudes. The radar is sufficiently sensitive to detect these signals above the noise threshold. Any significant forwardlooking data will overwhelm this signal during the daylight hours at higher latitudes due to gain bias towards that direction. The forward direction field-ofview is over the Sodankylä region, whilst the backward view is between Moscow and Riga. As we could not obtain ionosonde data for Riga we had to settle for the station at Uppsala. This is to the west of the CUTLASS field-of-view but is at a similar latitude. Interferometric information from the radars allows the elevation angle of the incoming signal path to be determined from which the look direction can be unambiguously deduced.

A method that is independent of the Finland radar for checking the look direction makes use of a combination of computational ray tracing and ionosonde data near the fields-of-view. The ionosondes provide information about the thickness and height of the electron density profile that are then input parameters to a model ionosphere. Radio wave ray paths are then computed for the Finland radar location and the range of the first detectable signal return established every hour. Figure 3 presents the results for January 11th, 1997, at Sodankylä, Uppsala and Moscow. Sodankylä produces a significant ionospheric propagation for fewer hours, compared with the longer periods for the more southerly locations and also results in a greater first skip distance that is not consistent with the CUTLASS observations for this month. The latter two exhibit a temporal displacement in line with their differing longitudinal positions.

The number of hours of daylight are closely related to the periods of significant power returns. However, changes in the ionosphere will be superimposed upon this. The Moscow ionosonde observed the electron density levels fall off quite noticeably around 12:00 UT and this was reflected in the CUTLASS power returns. Intervals have to be selected carefully where such events will not be present to contaminate the results and this usually means geomagnetically quiet conditions.

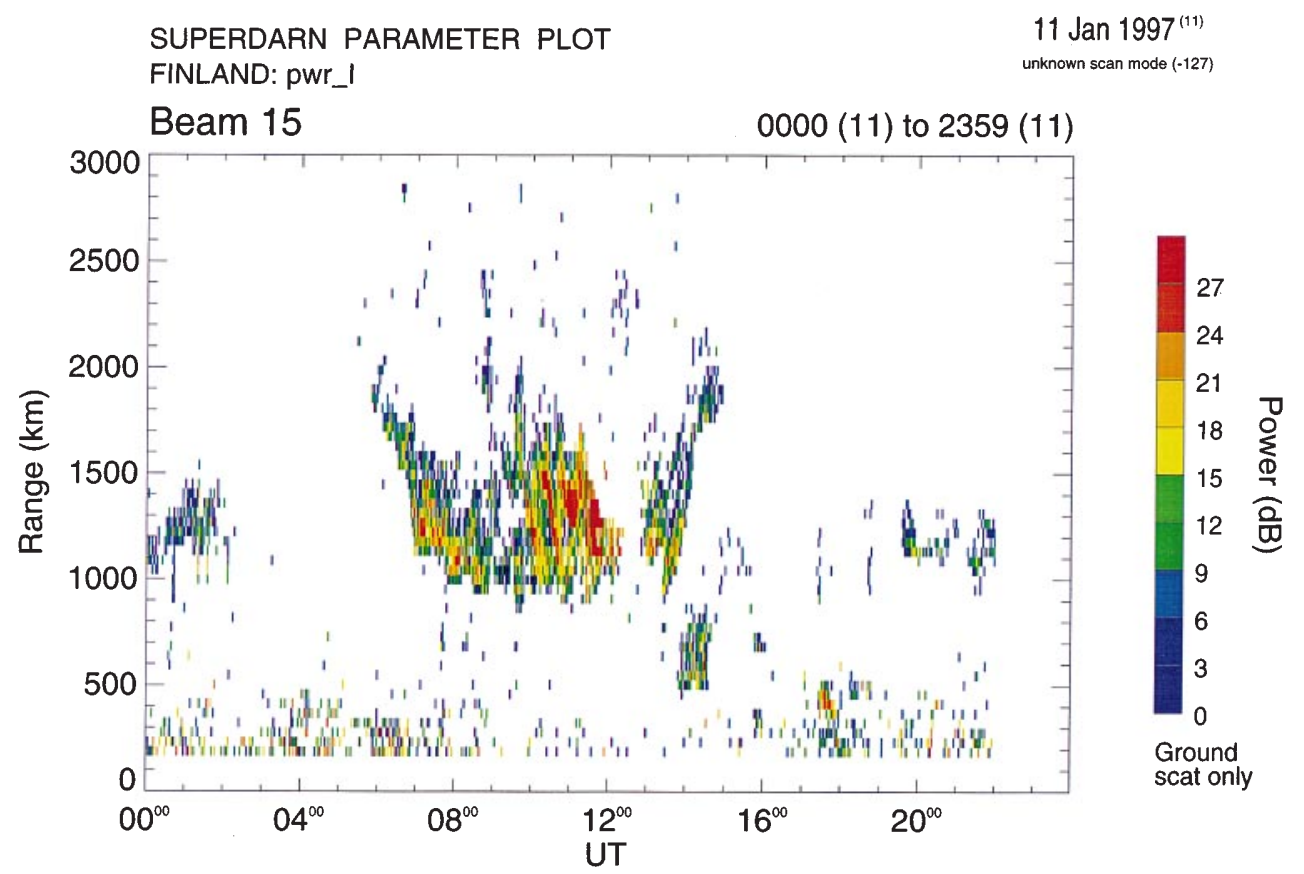

Fig. 2. Ground-scattered 'backward-looking' power returns from the Finland radar on the 11 January, 1997 for the beam closest to Moscow 


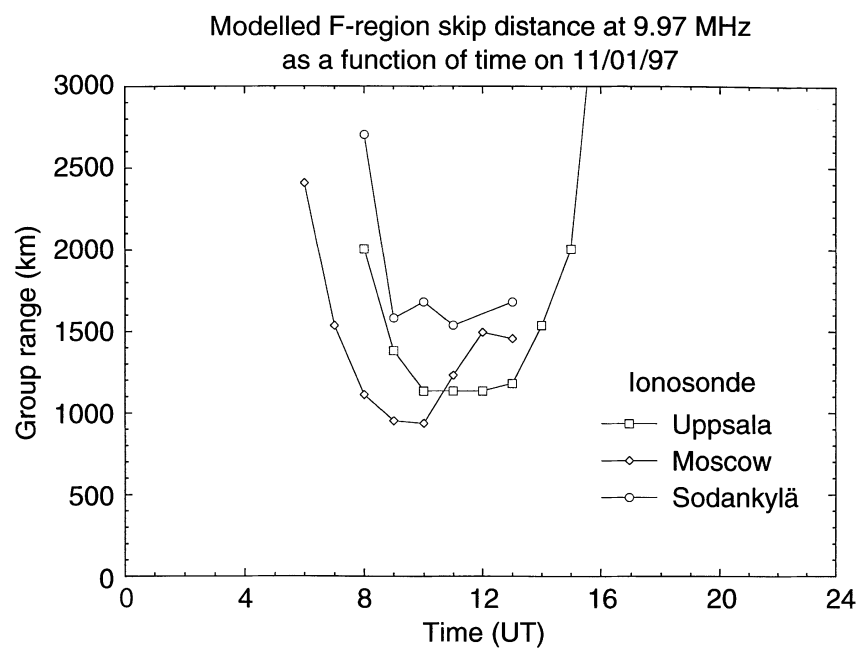

Fig. 3. Ray-tracing calculations of the F-region skip distance at $9.97 \mathrm{MHz}$ on 11/1/97 using ionospheric parameters obtained from the Sodankylä, Uppsala and Moscow ionosondes

\section{First return climatology and 'E-region de-contamination'}

To obtain the first ground-scatter returns from the F-region in the CUTLASS data set, it is necessary to discriminate against contamination from the E-region returns. This is often a simple task by visual inspection, but for routine data analysis, it is preferable to automate this activity. One means of accomplishing this is to compile a monthly climatology of first returns from the radar.
For every day of CUTLASS common programme observations and for each beam, the frequency of occurrence of the first power returns is noted as a function of range and time. The climatology for the Iceland data for January 1997 can be found in Fig. 4. Back-scatter returns have been ignored for the first ten range gates where geometrical considerations make it unlikely that the response is coming from the F-region. These range gates have thus been omitted from the figure. During daylight hours, the data corresponds to a smeared-out version of the ray-traced plot. The variability is caused by the passage of gravity waves and variations in the propagation conditions over the period of study. There is a small time shift for each beam which will produce added variability of the first returns, but this effect is small outside of the dawn/dusk periods where local time changes are far greater than TID displacements. E-region contamination is also quite noticeable in the near range gates. The first return statistics have been improved considerably by assuming that the F-region cannot be detected within the first 10 range gates. The only major difference between the Iceland and the Finland climatologies is a local time shift of four hours, which is consistent with the geographic separation of the two radars.

The mean range and first standard deviation at each time interval can then be computed. A five-point temporal filter smoothes out the variations caused by the relatively small statistical samples. The separation between the first standard deviations is of the order of 8 range gates. Any gravity wave disturbance from a typical day would be expected to generate F-region returns within this region. All observations more than
First hop stats Iceland January

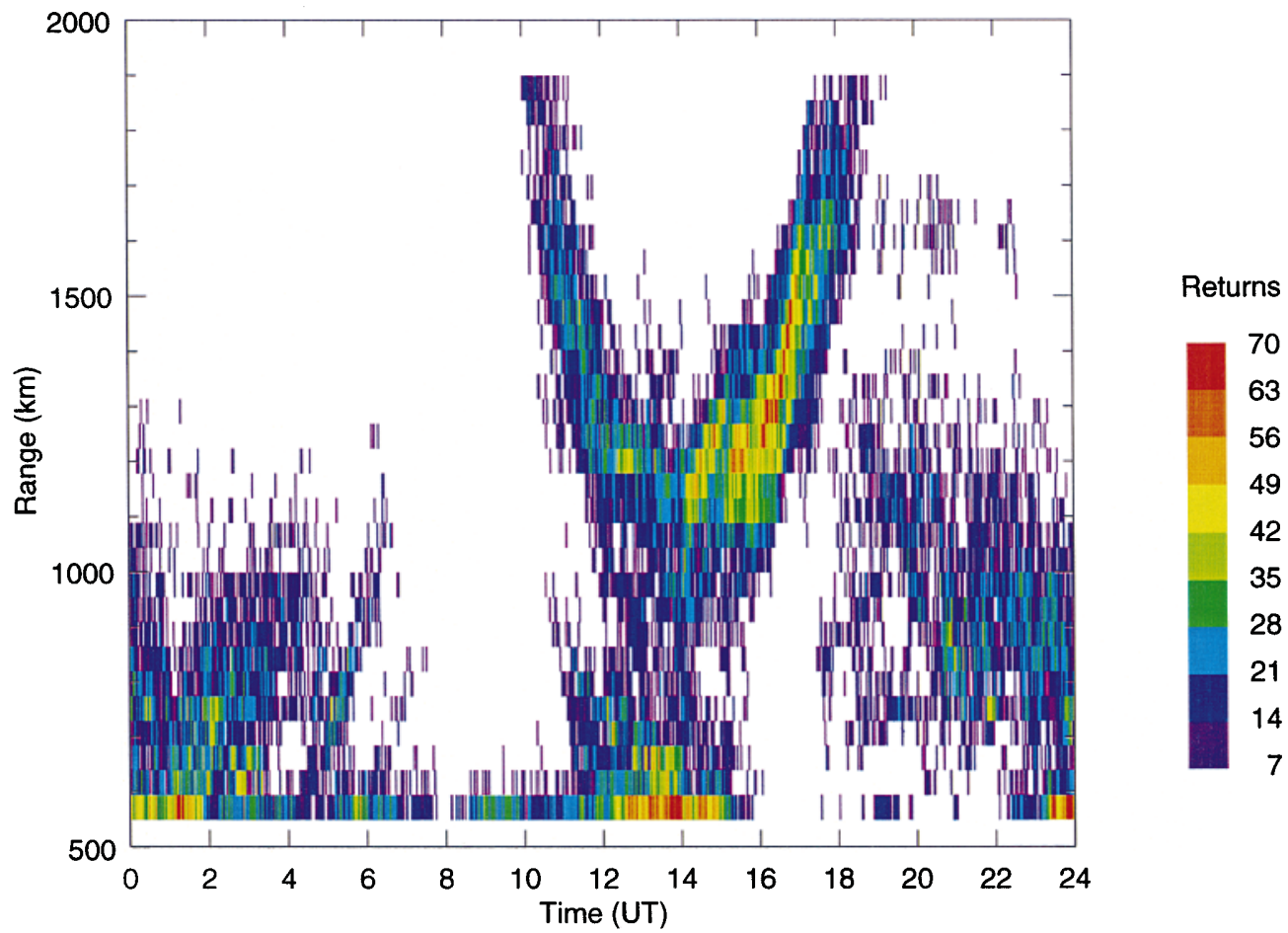

Fig. 4. First skip range statistics for January, 1997 combining all the beams from the Iceland CUTLASS radar with data from the first 10 range gates omitted 
one standard deviation from the mean can therefore be rejected.

Occasionally, the E-region first return is part of an extended area and so this filter can fail, producing contamination. Therefore, any data that is obtained at the nearest allowable range gate to the radar is considered to be unsound and no information from that sampling interval takes part in any further processing. A second stage of processing is then carried out using the filter outlined above for individual days. This procedure can then be repeated for the other 15 beams. A band pass filter can be applied to identify oscillations with periods of between 15 and $120 \mathrm{~min}$. An example from Iceland on January 8th, 1997, in Fig. 5 indicates that the waves are coherent across a number of beams, suggesting that the returns are likely to be from the same region of the ionosphere.

\section{Comparison between CUTLASS and EISCAT TIDs on 1/3/95}

The EISCAT facility comprises of a UHF system and a VHF radar. These wavelengths are ideal for measuring the scatter from acoustic waves inherent in the ionospheric plasma, rather than depending on coherent structures, as is the case for CUTLASS. However, the scattering cross section is very small and large power output is needed to compensate for this. Consequently, there are only a handful of such instruments in operation around the world and they do not run on a continuous basis.
We have selected a day when the EISCAT UHF instrument was running its Common Programme mode 1 , namely with a high temporal resolution in a single look direction along the geomagnetic field with good vertical resolution. Figure 6 shows the band-pass filtered electron density as a function of height and time on 1st March, 1995. The application of the filter makes these waves much more prominent. The disturbances tend to appear at a later time at lower altitudes, which is consistent with the downward propagating phase fronts and upward propagating energy of gravity waves. Previous studies have confirmed that these structures are closely related to AGWs (e.g. Shibata and Schlegel, 1993). Figure 7 shows the band-pass filtered electron density perturbations at $250 \mathrm{~km}$. The gravity waves change in amplitude and phase with height and so it is important to compare the correct EISCAT altitude with the effective reflection height of the observed CUTLASS data. Therefore, any technique that relies upon the reflection from the ionosphere would be height dependent. The peak in the EISCAT electron density profile on this day is $260 \mathrm{~km}$. CUTLASS has the ability to transmit at a range of frequencies between $8-20 \mathrm{~km}$ that can result in reflecting off different parts of the ionosphere, both height and range in a complex way that requires a combination of ray tracing and a comparison with EISCAT to fully understand.

The CUTLASS first hop climatology for March 1995 was constructed in the manner described in Sect. 4 and then the resulting E-region filter was applied to the first day of the month. Attention was

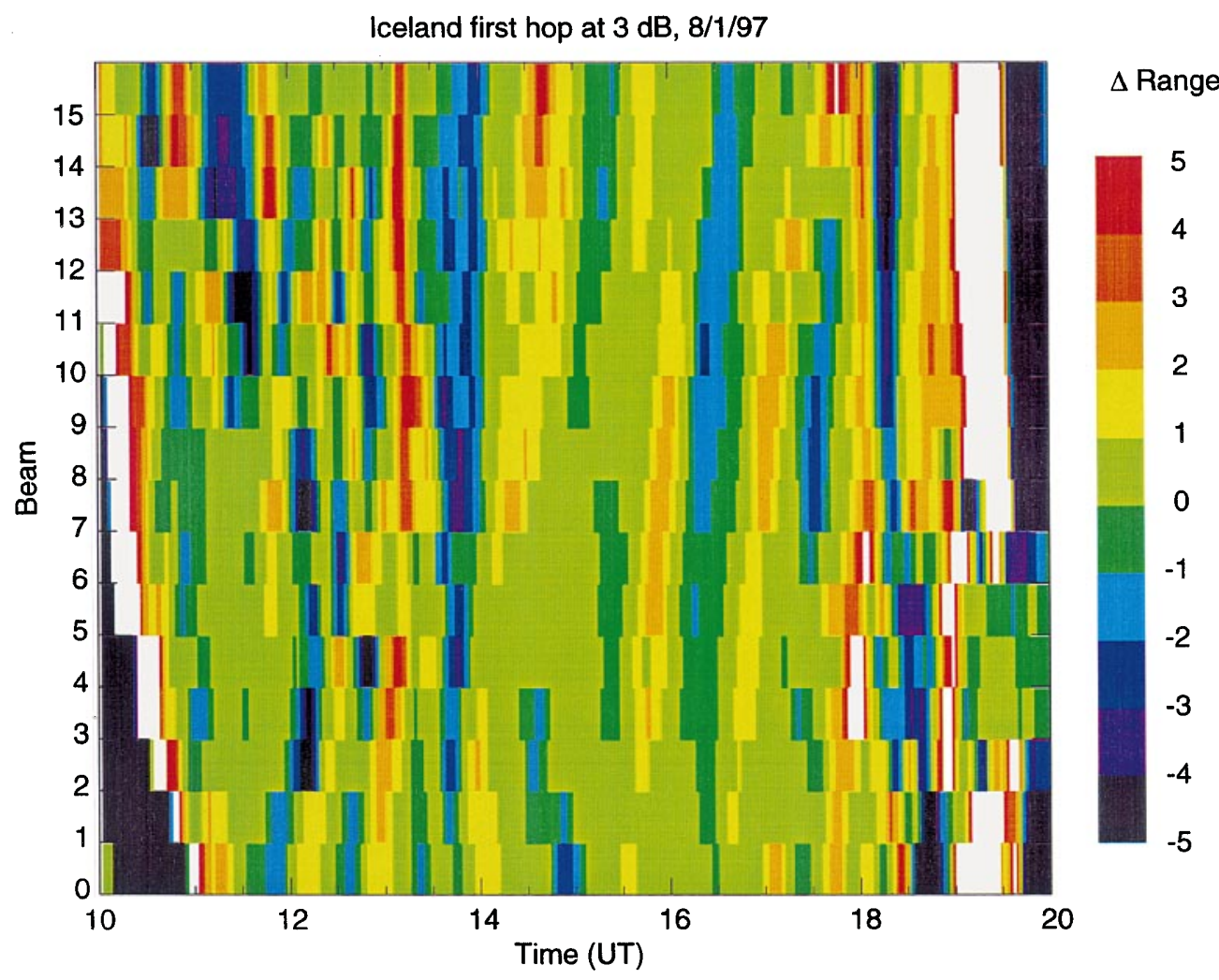

Fig. 5. Perturbations in the Iceland first returns after removing the diurnal variation for 8 January, 1997 across all 16 radar beams 


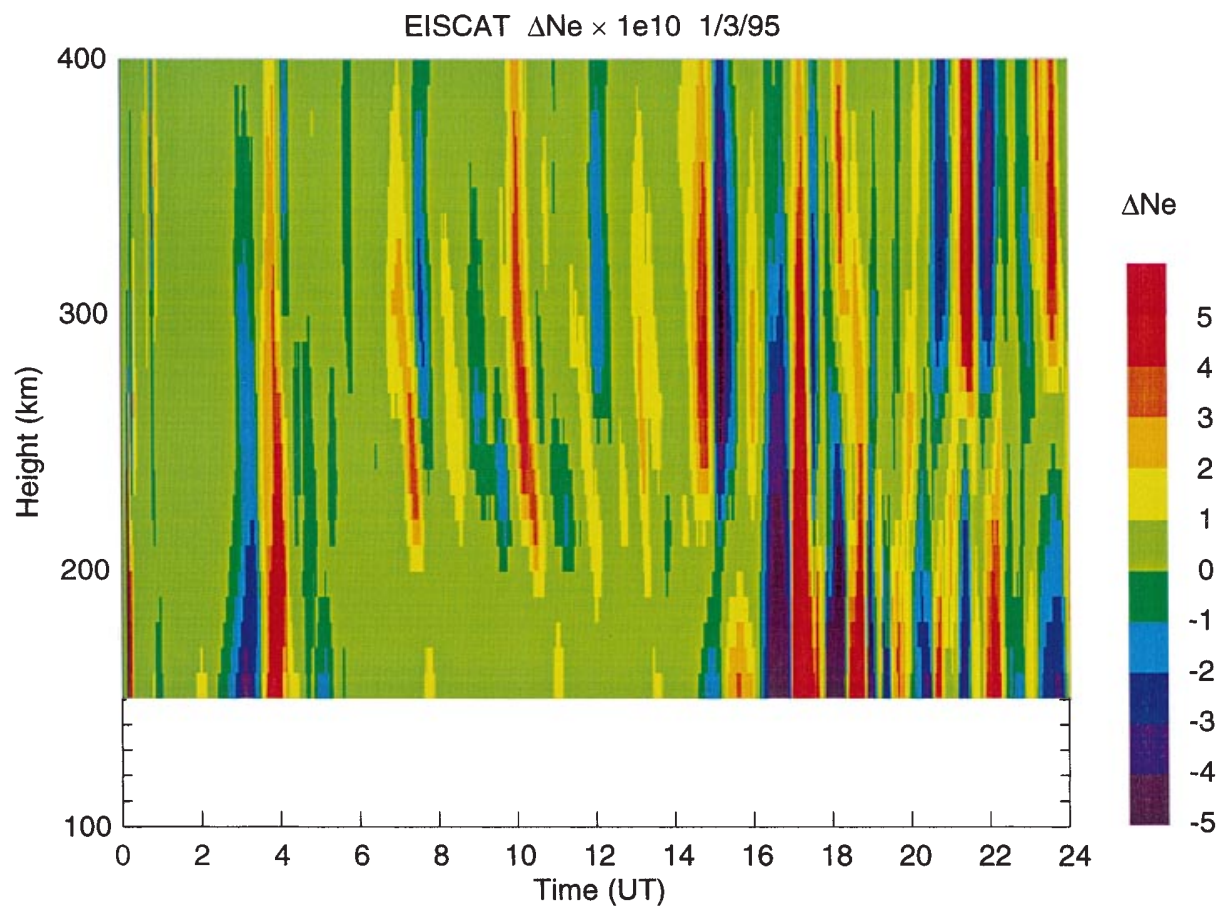

Fig. 6. Band-pass filtered electron density perturbations as a function of height and time from the EISCAT radar on 1 March 1995. The units are $10^{10} \mathrm{~m}^{-3}$

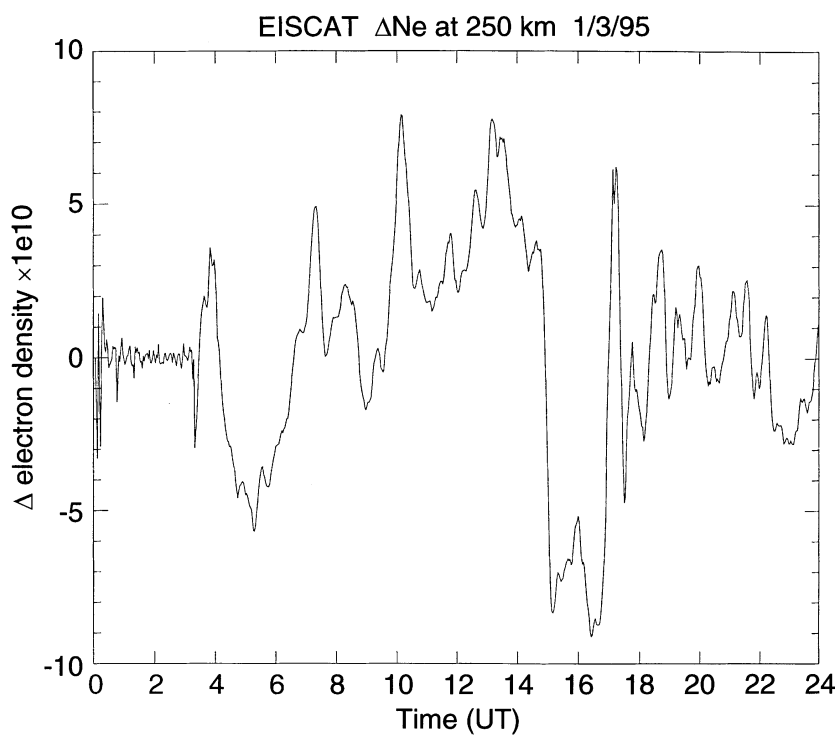

Fig. 7. Band-pass filtered electron density perturbations at a height of $250 \mathrm{~km}$ for 1 March, 1995

focused on beam 5 that looks towards the EISCAT radar site. There were a number of disturbances present in the daily first skip return that suggested strong gravity waves activity.

There appeared to be reasonable agreement between the CUTLASS and EISCAT time series. However, theoretical considerations indicate that for a maximum in electron density, the range should be at a minimum. This discrepancy may be accounted for by allowing a finite time for the wave that appears in the EISCAT radar field of view to reach the CUTLASS first return position. The range of the first CUTLASS returns for

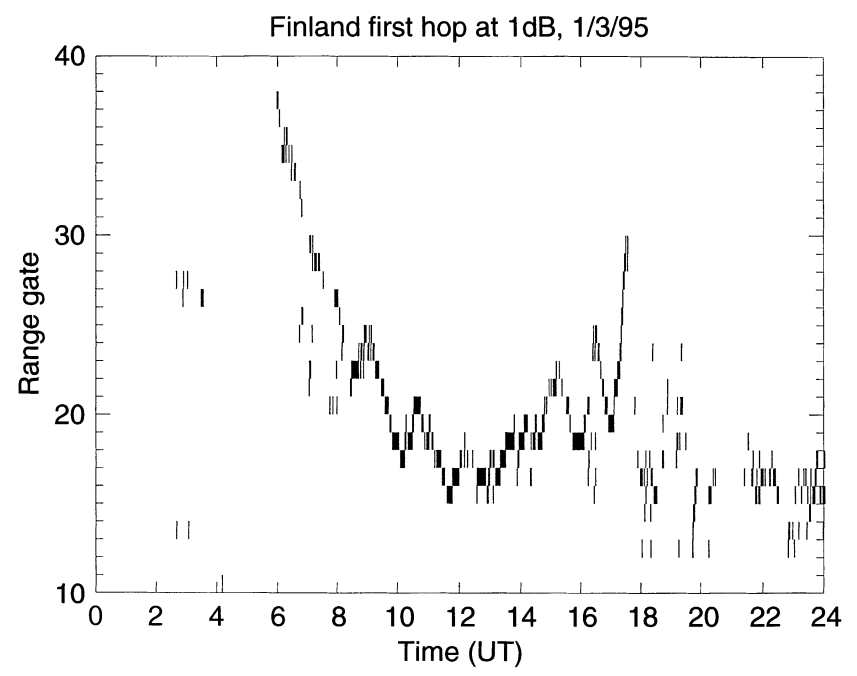

Fig. 8. Filtered first return data from CUTLASS Finland on 1 March, 1995 with the timing adjusted to allow for propagation for the Tromsø site assuming a line-of-sight phase velocity of $250 \mathrm{~ms}^{-1}$

1st March, 1995, are presented with a time delay added to take account of a delay for any wave to propagate from Tromsø to the CUTLASS field-of-view (Fig. 8). This time is proportional to the distance of the observations from Troms $\varnothing$ and assuming a constant line of sight phase speed of $250 \mathrm{~m} / \mathrm{s}$.

The correlation between the EISCAT and CUTLASS data for the interval 08:00 UT and 15:00 UT was computed to obtain the best agreement as a function of altitude and also of phase front speed. A correlation of -0.8 was obtained when EISCAT data at $235 \mathrm{~km}$ was used (Fig. 9) with a time delay of $108 \mathrm{~s}$ (Fig. 10). This is sufficiently below the peak electron density to assume 


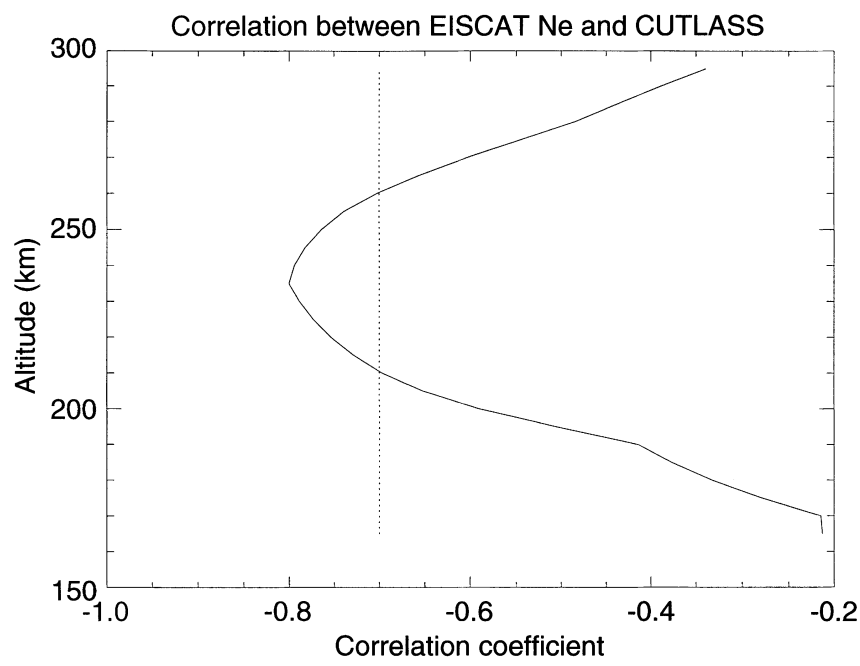

Fig. 9. Correlation coefficient between CUTLASS first range returns and the EISCAT electron density at a range of altitudes, assuming a delay of $108 \mathrm{~s}$ for the CUTLASS data

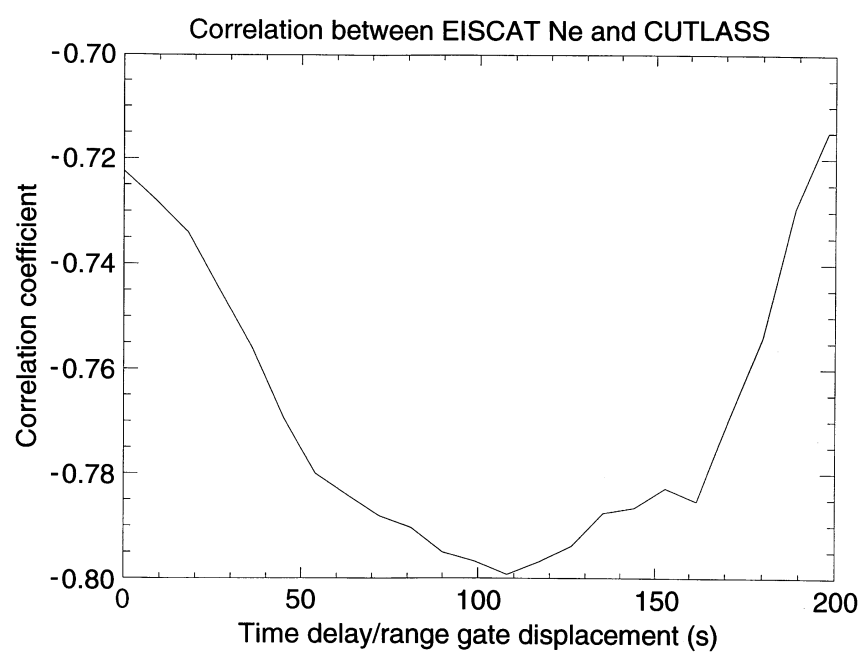

Fig. 10. Correlation coefficient between CUTLASS first range returns and EISCAT electron density at $235 \mathrm{~km}$, introducing a time delay in the CUTLASS data proportional to the distance from Tromsø

that any perturbations in the electron density field would produce a linear change in the altitude of the reflection point. The level of agreement is very good considering that two completely different measuring techniques were employed. No attempt was made at this stage to correct for the non-linear distortion discussed in Sect. 2 and the gravity wave field itself would have changed between the EISCAT and the CUTLASS first return positions. By assuming reflection occurs from the same height for each of the other 15 beams, electron densities over a $45^{\circ}$ arc may be derived. This would allow separation of purely temporal and spatial/temporal changes in the electron density field.

This result indicates that the technique can reproduce the general features of the electron density profile. Also, there is sensitivity to the perturbations due to the passage of gravity. Figure 10 shows that the correlation improved by 0.08 when the delay of $108 \mathrm{~s}$ was included in the CUTLASS data to allow for the propagation of the waves from Tromsø. As the ionospheric range is half the distance of the ground range, then each range gate represents a displacement of $22.5 \mathrm{~km}$ in the ionosphere. At an effective height of $235 \mathrm{~km}$, this produces a propagation speed of $210 \mathrm{~m} / \mathrm{s}$ towards the radar, consistent with previously reported measurements of medium-scale TIDs.

\section{Conclusions}

The CUTLASS radars are able to observe an extended region of the ionosphere from the ground backscattered power returns. Wave-like perturbations in the distance to the first F-region power returns are consistent with atmospheric gravity wave propagation. Variations in this distance give a good indication of the period of the waves and more uniquely, of their amplitude. The CUTLASS observations are consistent with a comparison with EISCAT data. We may therefore infer that we are seeing the signature of gravity waves in the CUTLASS radar skip distance signatures.

CUTLASS provides nearly continuous coverage of gravity waves over a wide area and a narrow height range, whilst the EISCAT radar gives very good height information at a single point (or three points in a different mode) on a few days every month. Therefore they can complement each other very well. In combination with the EISCAT Svalbard Radar, it will be possible to continue to monitor the evolution of waves from the polar cap ionosphere.

There are a number of ways to improve the experiment. The amplitudes of the atmospheric gravity waves can be inferred using a ray-tracing model that reproduces the expected range displacements. Information about the azimuthal orientation can be obtained from the details of the shape of the first returns. Also, it is possible to operate the radar at multiple frequencies with a constant scan rate by reducing the integration time proportionately. In this mode, one can probe the ionosphere at several separated regions from the radar. Information about the phase front speed of the disturbances towards the radar can then be inferred directly without reference to an external instrument such as EISCAT. The observational field of view improves considerably at the same time.

Iceland data became available from the middle of November, 1995, so further studies can make use of both CUTLASS radars after this date. Finally, increasing the horizontal resolution from $45 \mathrm{~km}$ to $15 \mathrm{~km}$, which is a proven feature of the instruments, will enhance the range sensitivity by a factor of three.

Acknowledgements. The authors wish to thank the staff at the Radio and Space Plasma Physics Group at Leicester for building and operating the CUTLASS radars. The Particle Physics and Astronomy Research Council of the United Kingdom funded this 
work. EISCAT is an international collaborative project funded by the UK, France, Norway, Japan, Sweden, Germany and Finland.

Topical Editor D. Alcaydé thanks W. Bristow and K. Schlegel for their help in evaluating this paper.

\section{References}

Arnold, N. F., and T. R. Robinson, A mechanism for generating non-linear electron density distributions when forced by large amplitude monochromatic gravity waves, Ann. Geophyicae., 12, 688-690, 1994.

Baron, M., The EISCAT facility, J. Atmos. Terr. Phys., 46, 469472, 1984

Bristow, W. A., and R. A. Greenwald, Estimating gravity wave parameters from oblique high-frequency backscatter: modeling and analysis, J. Geophys. Res., 100, 3639-3648, 1995.

Bristow, W. A., R. A. Greenwald, and J. C. Samson, Identification of high-latitude acoustic gravity wave sources using the Goose Bay HF radar, J. Geophys. Res., 99, 319-331, 1994.

Chimonas, G., and C. O. Hines, Atmospheric gravity waves launched by auroral currents, Planet. Space Sci., 18, 565-582, 1970.

Francis, S. H., A theory of medium-scale traveling ionospheric disturbances, J. Geophys. Res., 79, 5245-5260, 1974.

Georges, T. M., HF Doppler studies of travelling ionospheric disturbances, J. Atmos. Terr. Phys., 30, 735-746, 1968.

Gething, P. J. D., Radio direction finding and superresolution, Peter Perigrinus Ltd, London, pp 365, 1991.

Greenwald, R. A., K. B. Baker, J. R. Dudeney, M. Pinnock, T. B. Jones, E. C. Thomas, J.-P. Villain, J.-C. Cerisier, C. Senior, C. Hanuise, R. D. Hunsucker, G. Sofko, J. Koehler, E. Nielsen, R. Pellinen, A. D. M. Walker, N. Sato, and H. Yamagishi, DARN/SuperDARN: A global view of the dynamics of high-latitude convection, Space Sci. Rev., 71, 761796, 1995.

Hines, C. O., Internal atmospheric gravity waves at ionospheric heights, Can. J. Phys., 38, 1441-1481, 1960.

Hocke, K., and K. Schlegel, A review of atmospheric gravity waves and travelling ionospheric disturbances: 1982-1995, Ann. Geophysicae, 14, 917-940, 1996.

Hunsucker, R. D., Atmospheric gravity waves generated in the high-latitude ionosphere: a review, Rev. Geophys. Space Phys., 20, 293-315, 1982.

Kirchengast, G., K. Hocke, and K. Schlegel, Gravity waves determined by modeling of traveling ionospheric disturbances in incoherent-scatter radar measurements, Radio Sci., 30, 15511567, 1995.
Kirchengast, G., K. Hocke, and K. Schlegel, The gravity wave-TID relationship: insight via theoretical model-EISCAT data comparison, J. Atmos. Terr. Phys., 58, 233-243, 1996.

Lanchester, B. S., T. Nygrén, M. J. Jarvis and R. Edwards, Gravity wave parameters measured with EISCAT and dynasonde, Ann. Geophysicae, 11, 925-936, 1993.

Milan, S. E., T. B. Jones, T. R. Robinson, E. C. Thomas, and T. K. Yeoman, Interferometric evidence for the observation of ground backscatter originating behind the CUTLASS coherent HF radar, Ann. Geophysicae, 15, 29-39, 1997.

Millward, G. H., A resonance effect in AGWs created by periodic recurrent bursts in the auroral electric field, Ann. Geophysicae, 12, 94-96, 1994.

Munro, G. H., Travelling ionospheric disturbances in the $\mathrm{F}$ region, Aust. J. Phys., 11, 1958, 1958.

Natorf, L., K. Schlegel, and A. W. Wernik, Gravity wave parameters derived from travelling ionospheric disturbances observations in the auroral zone, Radio Sci., 27, 829-840, 1992.

Robinson, T. R., Acoustic gravity wave growth and damping in convecting plasma, Ann. Geophysicae, 12, 210-219, 1994.

Samson, J. C., R. A. Greenwald, J. M. Ruohoniemi, A. Frey, and K. B. Baker, High-frequency radar observations of atmospheric gravity waves in the high-latitude ionosphere, Geophys. Res. Lett., 16, 875-878, 1989.

Samson, J. C., R. A. Greenwald, J. M. Ruohoniemi, A. Frey, and K. B. Baker, Goose Bay radar observations of Earth-reflected atmospheric gravity waves in the high-latitude ionosphere, J Geophys. Res., 95, 7693-7709, 1990.

Schlegel, K., The study of tides and gravity waves with the help of the field-aligned velocities measured by EISCAT, J. Atmos. Terr. Phys., 48, 879-886, 1986.

Shibata, T., and K. Schlegel, Vertical structure of AGW-associated ionospheric fluctuations in the E- and lower F-region observed with EISCAT - case study, J. Atmos. Terr. Phys., 55, 739-749, 1993.

Tedd, B. L., H. S. Strangeways, and T. B. Jones, The influence of large-scale travelling ionospheric disturbances on the bearings of geographically spaced HF transmissions, J. Atmos. Terr. Phys. 46, 109-118, 1984.

Tedd, B. L., and M. G. Morgan, TID observations at spaced geographic locations, J. Geophys. Res., 90, 2307-2319, 1985.

Williams, P. J. S., T. S. Virdi, R. V. Lewis, M. Lester, A. S. Rodger, I. W. McCrea, and K. S. C. Freeman, 'Worldwide atmospheric gravity-wave study in the European sector 1985-1990', J. Atmos. Terr. Phys., 55, 683-696. 